\title{
Evaluation of Autonomic Nervous Activity Based on Arterial Wall Impedance and Heart Rate Variability
}

\author{
Abdugheni KUTLUK $^{1}$, Kenji SHIBA ${ }^{1}$, Toshio TSUJI ${ }^{1}$ \\ Ryuji NAKAMURA ${ }^{2}$, Noboru SAEKI ${ }^{2}$, Masashi KAWAMOTO ${ }^{2}$ \\ ${ }^{1}$ Graduate School of Engineering, Hiroshima University, \\ Higashi-Hiroshima, Japan 739-8527 \\ ${ }^{2}$ Graduate School of Biomedical Sciences, Hiroshima University, \\ Hiroshima, Japan 734-8551 \\ akutluk@bsys.hiroshima-u.ac.jp
}

\begin{abstract}
This paper proposes a new method for evaluating the autonomic nervous activities by using the mechanical impedance of the arterial wall and heart rate variability. The cardiovascular system is indispensable to life maintenance functions, and homeostasis is maintained by the autonomic nervous system. It is very important to give a diagnosis based on the autonomic nervous activities of the whole body. The proposed method is evaluated in surgical operations, where the mechanical impedance of the arterial wall is estimated from the arterial blood pressure and a pulseoximetric photoplethysmogram, and heart rate variability is estimated by electrocardiogram $R R$ interval spectral analysis. From the experimental results, we concluded that the proposed method is able to estimate autonomic nerve activities corresponding to events during operations.
\end{abstract}

Keywords-Mechanical impedance, Autonomic nerves, Arterial wall, Heart rate variability, Photoplethysmogram.

\section{INTRODUCTION}

In medical treatment sites, a doctor needs to accurately judge the patient's autonomic nervous conditions and take appropriate measures. Generically, doctors used to judge patient's conditions during operations from vital signals and measures such as electroencephalogram (EEG), electrocardiogram (ECG) and blood pressure (BP) [1]. However, doctors must have expert knowledge and sufficient experience to catch subtle changes in wave forms, infer causes, and make appropriate judgment. Particularly, in the case of emergency situations or during surgery, if a monitoring support system for cardiovascular conditions becomes available, the medical staff will be able to more easily and quickly identify patient's conditions.

The cardiovascular system is indispensable to life, and homeostasis is maintained by the autonomic nervous system (ANS). It is known that one part of the ANS based on heart activities is reflected in fluctuations of the heart rate [2]. Many studies to date have been proposed for heart rate variability (HRV) by frequency domain analysis and time domain analysis [3-6]. Recent studies have also used power spectrum analysis to evaluate ANS activities from changes in the blood pressure and heart rate during the evaluation exercise [7-8]. However, these studies evaluated with only ANS activities appearing in the activity of the heart, and did not sufficiently evaluate the entire cardiovascular system.

On the other hand, some researchers have tried to describe the detailed characteristics of vascular smooth muscles, wherein vascular tone (hardness) represents an index of ANS activity of the arterial wall [9-11]. Also, applying the skeletal muscle impedance estimation method [13], the authors modeled the dynamic characteristics of the human arterial wall by employing mechanical impedance, including stiffness, viscosity and inertia to estimate the changing beat-to-beat conditions of blood vessels and ascertained the vascular conditions in the physician's surgical actions as impedance changes [12-15]. However, the developed system only evaluated the ANS activity of the peripheral vessel, and was not enough to understand the autonomic nervous activity of the entire cardiovascular system.

The present paper proposes a new monitoring system to comprehensively evaluate ANS activity on the cardiovascular system by simultaneously analyzing the arterial wall impedance and the HRV, and also displaying the bispectral index (BIS) estimated from EEG. In the proposed system, the ANS activity of the heart is estimated by HRV, and the ANS activity of blood vessel is estimated by arterial wall impedance. The proposed system aims to develop an on-line monitoring (support) system in surgical operations, which can estimate ANS activity from beat-to-beat. Using the proposed system, we monitor ANS activity during endoscopic transthoracic sympathectomy surgery and assess the validity of the proposed method.

\section{COnfiguration OF MONitoring System}

A structure of the proposed diagnosis ANS activity support system is shown in Fig. 1. The proposed system consists of three parts, I. Signal measurement, II. Parameter estimation, III. Display. In the monitoring support system, parts II and III are constructed with a graphical display screen using LabVIEW (National Instruments Corp.). The methods of preprocessing and estimating ANS activity used in a prototype are described below. 


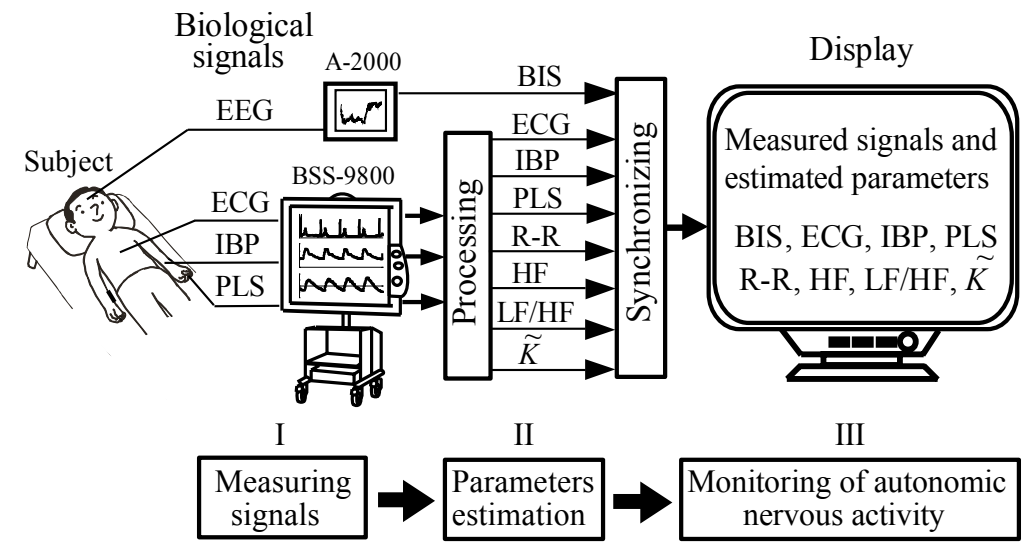

Fig. 1 System configuration

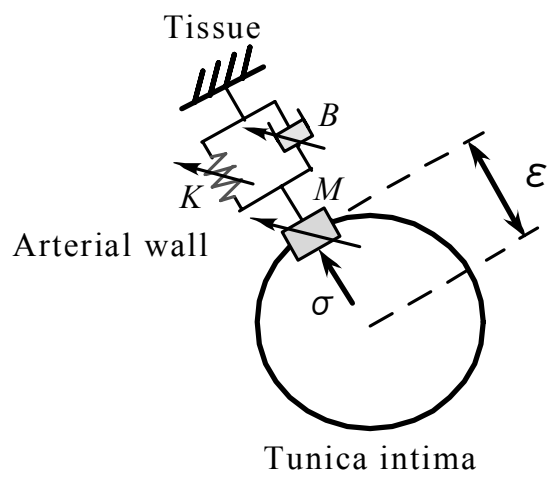

Fig. 2 The arterial wall impedance model

\section{A. Signal Measurement}

ECG, BP and a pulseoximetric photoplethysmogram (PLS) are measured at $125 \mathrm{~Hz}$ simultaneously and stored on a personal computer by using Transmission Control Protocol (TCP). The BP is measured through a catheter (24 gauge) placed in the left radial artery, and the PLS is measured with the ipsilateral thumb (BSS-9800, NIHON KOHDEN Corp.). The bispectral index (BIS) was recorded using the Aspect A2000 EEG monitor (Aspect Medical Systems Inc.) and BIS values were transferred in 5 second intervals and stored on a personal computer.

\section{B. Estimation and Display of Autonomic Nervous Activity}

\section{1) Estimation of Arterial Wall Impedance:}

For extracting vascular features included in the measured signals, the arterial wall impedance is estimated [14]. Fig.2 illustrates the proposed impedance model of the arterial wall. This model represents only the characteristics of the arterial wall in the arbitrary radius direction. The impedance characteristic can be defined as a relation between an exerted force and a displacement of the arterial wall, expressed in the following equation:

$$
d \sigma(t)=M d \ddot{\varepsilon}(t)+B d \dot{\varepsilon}(t)+K d \varepsilon(t)
$$

where $\sigma(t)$ is the stress exerted on the arterial wall by blood pressure; $M, B$, and $K$ represent the inertia, viscosity, and stiffness; $\varepsilon(t), \dot{\varepsilon}(t)$, and $\ddot{\varepsilon}(t)$ are the strain, strain velocity, and strain acceleration of the wall; the coefficient $d$ means the variation from the time $t_{0}, d \sigma(t)=\sigma(t)-\sigma\left(t_{0}\right), d \varepsilon(t)=\varepsilon(t)-\varepsilon\left(t_{0}\right)$; and $t_{0}$ denotes the time when the $\mathrm{R}$ wave appeared in each ECG recognition cycle [15].

To estimate the impedance parameters given in equation (1), it is necessary to measure $\sigma(t)$ and $\varepsilon(t)$. Assuming that the stress $\sigma(t)$ is proportional to the direct arterial pressure $P_{b}(t)$, the following equation can be obtained:

$$
\sigma(t)=k_{f} P_{b}(t)
$$

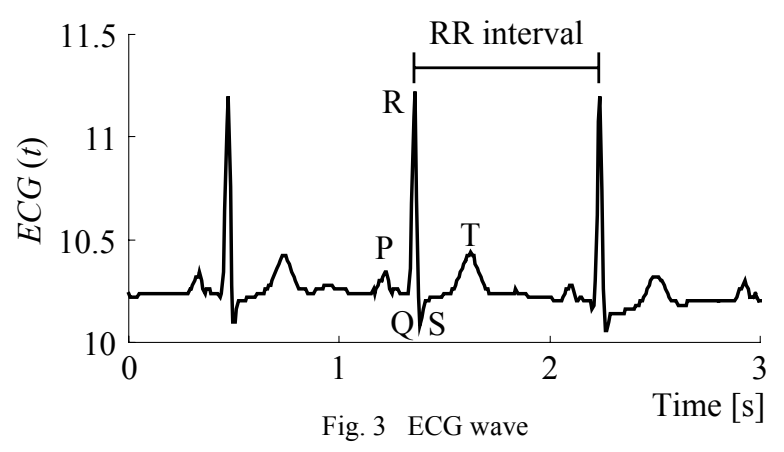

where $k_{f}$ is a proportional constant. On the other hand, the vascular strain $\varepsilon(t)$ is quite difficult to measure directly. Then, a plethysmogram is utilized instead of $\varepsilon(t)$ as follows:

$$
\varepsilon(t)=k_{p} P_{l}(t)
$$

where $P_{l}(t)$ is the measured plethysmogram, and $k_{p}$ is a proportional constant [8].

The stress exerted on the arterial wall is expressed by the direct arterial pressure $P_{b}(t)$ given by equation (2), and the strain of the arterial wall $\varepsilon(t)$ is represented by the plethysmogram $P_{l}(t)$ in (3). It has been shown that inertia $M$ is very small and can be ignored in the transversal arteries [16]. Then, the arterial wall impedance parameters can be estimated by using the least square method from the measured signals $P_{b}(t)$ and $P_{l}(t)$, which gives rise to following equation:

$$
d P_{b}(t)=\widetilde{B} d \dot{P}_{l}(t)+\widetilde{K} d P_{l}(t)
$$

where $\quad d P_{b}(t)=P_{b}(t)-P_{b}\left(t_{0}\right) ; \quad d P_{l}(t)=P_{l}(t)-P_{l}\left(t_{0}\right)$; $d \dot{P}_{l}(t)=\dot{P}_{l}(t)-\dot{P}_{l}\left(t_{0}\right) ; d \ddot{P}_{l}(t)=\ddot{P}_{l}(t)-\ddot{P}_{l}\left(t_{0}\right) . \widetilde{B}$ and $\widetilde{K}$ correspond to the viscoelastic properties of the arterial wall respectively. 


\section{2) Heart Rate Variability Estimation:}

Heart rate rhythm is adjusted by ANS activity. The variation of beat-to-beat intervals referred to heart rate variability (HRV), can usually be calculated by analyzing the time series of R-R intervals from ECG in Fig. 3. Various HRV spectral analyses have been proposed using the Fast Fourier Transform (FFT) and Wavelet Transform (WT) analysis [3-5]. The principle of spectral analysis is based on the fact that the sympathetic nervous system (SNS) and parasympathetic nervous system (PNS) activities reflect on the heart rate (R-R interval) variability in specific frequency bands. Generally, it is known from the blocking drugs experiment of the nervous system that the low frequency components (LF: $0.04 \sim 0.15 \mathrm{~Hz}$ ) reflect both SNS and PNS activity, and that the high frequency components (HF: $0.15 \sim 0.5 \mathrm{~Hz}$ ) reflect the PNS activity. The ratio LF/HF reflects the SNS activity [4-5].

In this study, the cardiac ANS activity is estimated by using WT (high time resolution) based power spectral analysis, and $\mathrm{HF}$ and LF/HF components are computed. We utilized Fluclet software (Dainippon-Sumitomo pharma Co. Japan) for the HRV analysis [6].

\section{3) Display:}

The display of the developed system is given in Fig. 4. In the upper left portion of the screen, the measured electrocardiogram (ECG), invasive arterial pressure (IBP) and photoplethysmogram (PLS) are shown as the measured biological signals. The upper right portion of the screen shows the Lissajous figure, where the of a horizontal axis is the IBP wave and the vertical axis is the PLS wave, so that the inclination of the Lissajous figure, which is the ratio of the amplitudes of the plethysmogram and arterial pressure, gives an approximation of blood vessel compliance [9]. At the bottom of the screen, the estimated 5 parameters (BIS, R-R, HF,
LF/HF, $\widetilde{K}$ ) represent an index of awakening of the brain and the ANS activity of the heart and arterial wall. In this system, the changes in a patient's ANS activity on the cardiovascular system can be understood both visually and easily. The medical doctors can predict whether a patient's condition will take a turn for the better or become more and more dangerous from the changes in the signals.

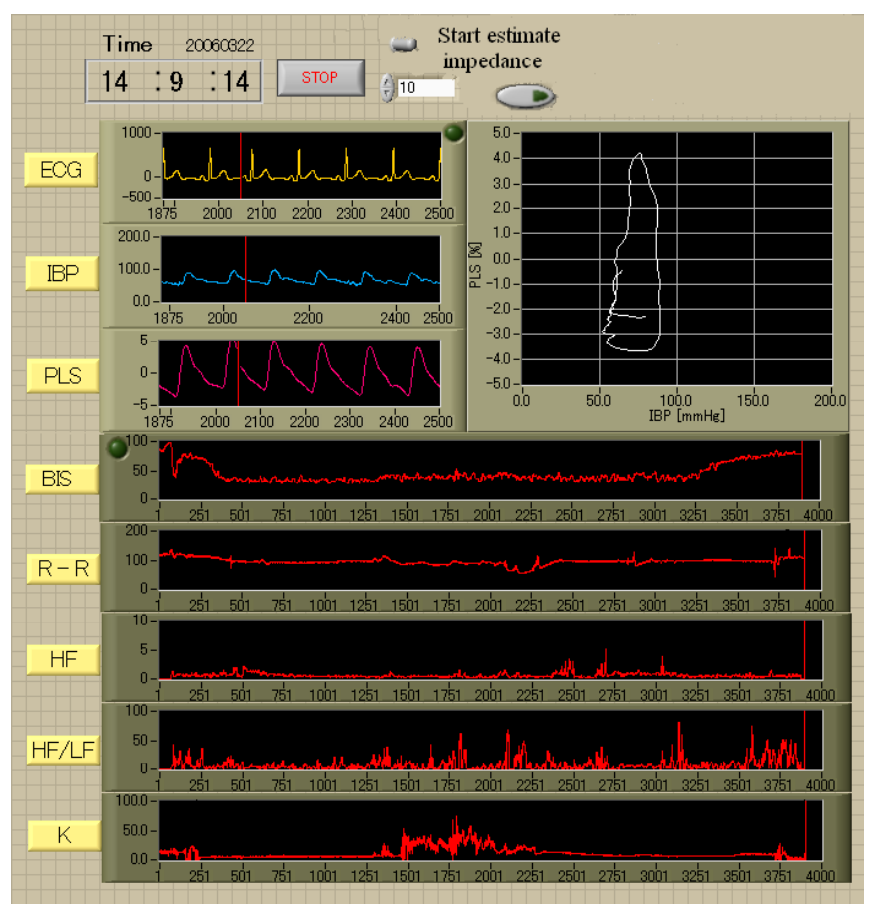

Fig. 4 The parameters on the display screen image

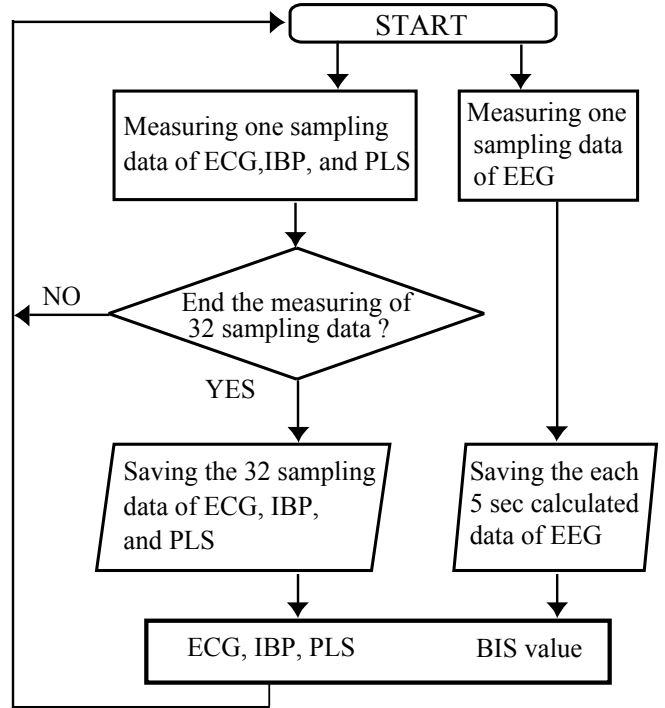

(a) Measuring the biological signals

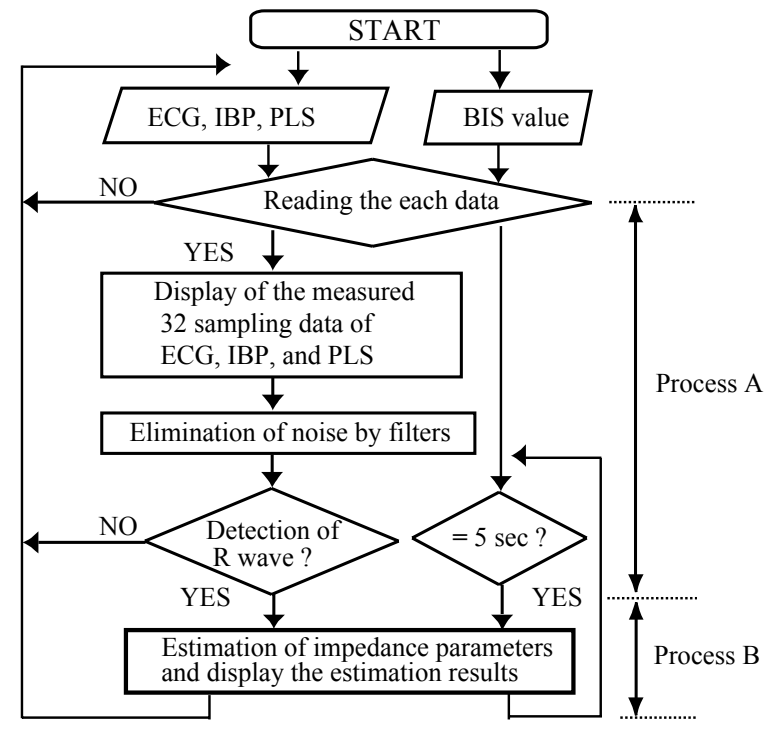

(b) Signal processing

Fig. 5 Flowchart of the signal processing 


\section{4) Process Flowchart:}

Fig. 5(a) explains the measuring of the biological signal for communication of the personal computer and the bedside monitor. The bedside monitor measures the electrocardiogram, arterial pressure, and photoplethysmogram simultaneously (Fig. 5(a)). The sampling time is $8 \mathrm{~ms}$. The measured biological signals are stored in the bedside monitor and the data are transferred all at once using TCP when 32 sampling data are stored. The transfer time is about $0.2 \mathrm{~ms}$, which is sufficiently short compared with the sampling time. Fig. 6 demonstrates an example of the measured biological signals. This figure plots the electrocardiogram, arterial pressure, and photoplethysmogram. The BIS value is calculated by the EEG monitor, which are transferred in every 5 seconds and stored on a personal computer.

Fig. 5(b) explains the estimation of parameters on the personal computer. The measured biological signals are read in every 32 sampling data, and then displayed in the upper left portion of the screen (process A). Because the data are affected by some artifacts, such as light and mechanical stimulation on the patient's hand, the arterial pressure and photoplethysmogram are preprocessed using digital filters. The invasive arterial pressure were filtered out through a second-order infinite impulse response (IIR) lowpass filter with the cutoff frequency of $6 \mathrm{~Hz}$ and a first-order IIR high-pass filter with a cutoff frequency of $0.3 \mathrm{~Hz}$, respectively, and the photoplethysmogram is filtered through an eighth-order finite impulse response (FIR) low-pass filter with the cutoff frequency of $15 \mathrm{~Hz}$ and a first-order IIR high-pass filter with the cutoff frequency of $0.3 \mathrm{~Hz}$. After the filtering process, the $\mathrm{R}$ wave in each ECG recognition cycle is detected (process $\mathrm{A}$ end). If the $\mathrm{R}$ wave is detected, then processing is begun to estimate (process B) each parameter (R-R, HF, LF/HF, $\widetilde{K}$ ). When the $\mathrm{R}$ wave is detected, the arterial pressure and plethysmogram, which are measured data from the previous $\mathrm{R}$ wave to the detected $\mathrm{R}$ wave, are substituted in equation (4) and the impedance parameters are estimated. Because the previous $\mathrm{R}-\mathrm{R}$ interval is established each time an $\mathrm{R}$ wave is detected, the beat-to-beat arterial wall impedance parameters $\widetilde{K}$ can be estimated. The estimation results are displayed at the bottom of the screen (results $\widetilde{K}$ ) in Fig. 4. At the same time, wavelet transform (high time resolution) based HRV analysis is used to estimate the cardiac ANS activity (R-R, HF, LF/HF). The estimation results are displayed at the bottom of the screen (results R-R, HF, LF/HF) in Fig. 4 (process B end).

As for the estimation time of the parameters (BIS, R-R interval, HF, LF/HF, $\widetilde{K}$ ) showed in Fig.4, R-R and $\widetilde{K}$ are renewed from beat-to-beat, and parameters BIS, HF, LF/HF are synchronized every second and then renewed on the screen.

The medical doctors can identify whether the patient's condition is stable from the biological signals, arterial wall impedance and cardiac ANS activity. Also, they can be judged whether anesthesia is adequately taking effect or not.

\section{MONITORING EXPERIMENT}

To evaluate the validity of the proposed method, the ANS activity during the surgical operations was evaluated by using
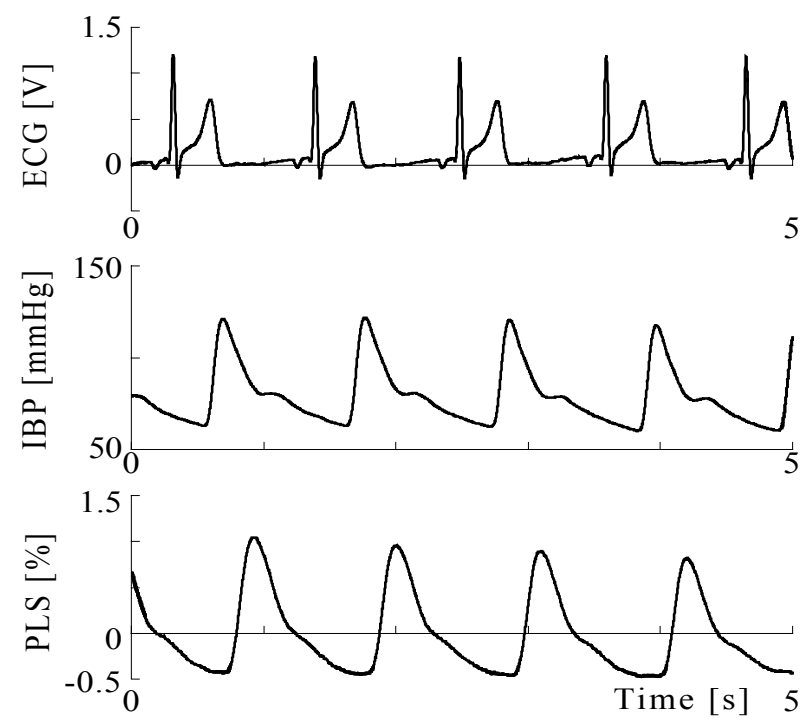

Fig. 6 Examples of the biological signals

off-line analysis. The proposed system aims towards an on-line monitoring that can be applied to surgical operations. As a halfway stage of system development, the biological signals measurement was taken on-line, and the signals analysis was performed taken off-line. The operations on the endoscopic transthoracic sympathectomy for hyperhidrosis with four patients were used for off-line analysis. The endoscopic transthoracic sympathectomy is a surgery for hyperhidrosis patients, whose sympathetic nerves are in a hyperfunction state, and the patients perspire copiously in the palms and armpits compared to ordinary people. In this operation, the sympathetic nerve running along the spine is clipped to stop perspiration [17]. When the sympathetic nerve is clipped, it is expected the blood vessels become compliant on the spot.

In this operation, ECG, BP, PLS, EEG are measured simultaneously by using the ipsilateral thumb and EEG monitor. The BP was measured through a 24 gauge catheter placed in the left radial artery, and the PLS was measured from both the left and right thumbs. A personal computer LATITUDE/610 (DELL, Pentium IV 2.0GHz) was used for the monitoring system.

\section{RESUlTS AND DisCUSSION}

In order to assess the validity of the proposed method, the data of four patients (patient A, B, C, D) with correct interruption of the sympathetic nerves were analysed. Fig. 7 shows an example of experimental results of the estimated ANS activities during surgery. In order from the top, the BIS, $\mathrm{R}-\mathrm{R}$ interval variability, $\mathrm{HF}, \mathrm{LF} / \mathrm{HF}$, and the peripheral ANS activities $\widetilde{K}$ are described. The right and left side sympathetic nerves of the patient were clipped at the dotted lines A and B. In the figure, the timing of the induction and the cessation (wearing off) of anesthesia are indicated as (I) and (IV). The surgeon stimulated the patient's tissues by identifying the sympathetic nerve from right to the left at the area (II) and (III). 
It can be seen that, as for the timing on the induction of anesthesia (I) and cessation of anesthesia (IV) area, the sympathetic nerves activities (LF/HF) decreased and increased, respectively. When these painful stimuli were applied $\left(\mathrm{E}_{1}, \mathrm{E}_{2}\right)$, peripheral vascular tone $\widetilde{K}$ increased because sensory information was transmitted from the medulla spinalis to the brain, and peripheral vasoconstriction and muscle contraction occurred. After the sympathetic nerve was clipped, the blood vessels became markedly compliant $\left(\mathrm{F}_{1}, \mathrm{~F}_{2}\right)$ and $\widetilde{K}$ decreased because sympathetic nerve activities could not be transmitted to the peripheral part. Furthermore, it can be understood that because the surgeon stimulated and clipped the patient's sympathetic nerve. It should be noted that the LF/HF activities increased before the clipping $\left(\mathrm{C}_{1}, \mathrm{C}_{2}\right)$ and after the clipping $\left(\mathrm{D}_{1}, \mathrm{D}_{2}\right)$.

Fig. 8 shows the comparative results of the mean values and the standard deviations for 4 patients in 180 seconds of data on the resting (normal) state (i), before the clipping (ii) on the right side, after the clipping (iii) on the right side, before the clipping (iv) on the left side, and before the clipping (v) on the left side. Because the sympathetic nerve was clipped adequately, after the clipping ((iii) and (v)) $\widetilde{K}$ recovered to the resting levels (i) after the nerve was clipped. Also, it can be understood from the HRV results that LF/HF activities are increased from (ii), (iv) to (iii), (v). This could be caused by the clipping, the effects of which appeared in the cardiac ANS activity.

Consequently, it can be seen that, from LF/HF and $\widetilde{K}$, we can monitor the patient's cardiac and peripheral autonomic nervous activities. It can also be seen that anesthesia depth was obtained based on comparison of LF/HF and $\widetilde{K}$ with the BIS index.

\section{CONCLUSIONS}

We have developed a monitoring system for estimating ANS activity by simultaneously analyzing the arterial wall impedance and the heart rate variability, and have ascertained the validity of the proposed method during the surgery. Our results show that the estimated ANS activities correspond to the events during operations.

Future research will be directed to test the online version of the proposed system and the number of subjects will be increased. It will also be ascertained for a wide variety of uses in the general environment.

\section{ACKNOWLEDGMENT}

The authors would like to thank Mr. T.Ukawa of the NIHON KOHDEN Co., Ltd for making available clinical data for this research. This work was supported by the knowledge Cluster for Scientific Research of Japan Society for the Promotion of Science.

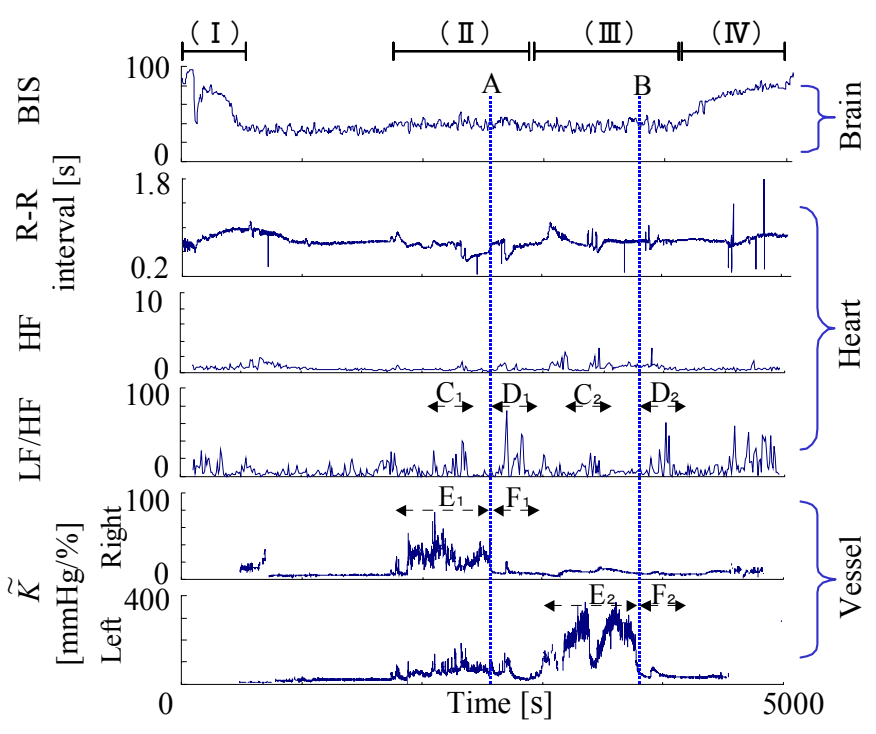

Fig. 7 Examples of the estimated parameters (Patient A)

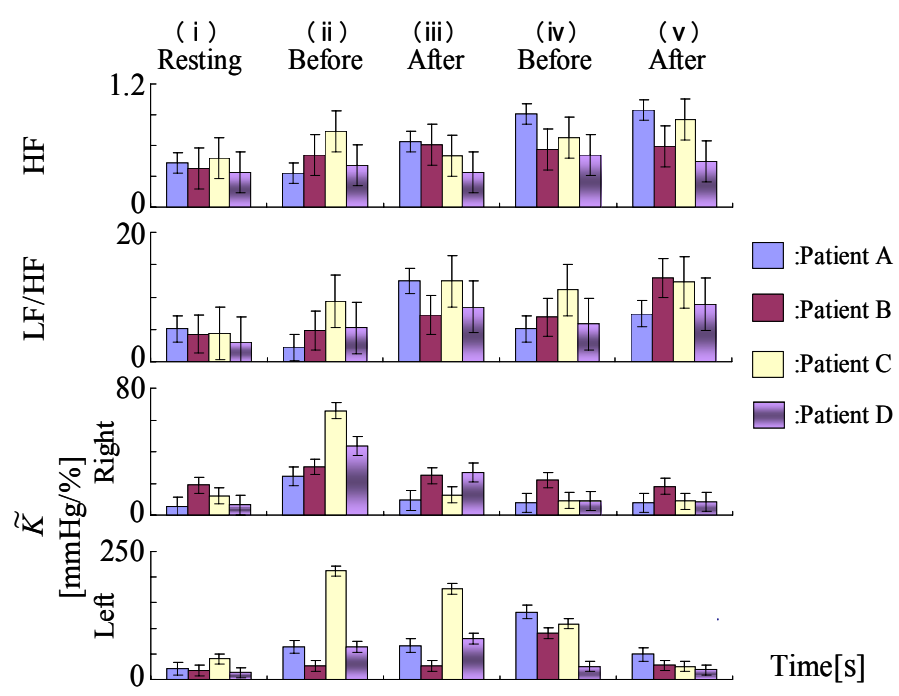

Fig.8 Estimated results of autonomic nervous activities

\section{REFERENCES}

[1] T.Kumazawa, O.Yugi, H.Furuya, "Standart anesthesia," pp. 220-231, 2002, (in Japanease).

[2] H.Inoue, "Cardiovascular disease and autonomic nervous function", pp. 5-10, 2001, (in Japanease).

[3] Akselrod.S, Gordon.D, Ubel.FA, Shannon.DC, Berger.AC, Cohen.RJ, "Power spectrum analysis of heart rate fluctuation: a quantitative probe of beat-to beat cardiovascular control," Science, Vol. 10, pp. 220-222, 1981.

[4] Yamamoto.Y, Hughson.RL, Peterson JC, "Autonomic control of heart rate during exercise studied by heart rate variability spectral analysis," $J$ Appl Physiol, Vol. 71, pp.1136-1142, 1991.

[5] Task Force of the European Society of Cardiology and the North American Society of Pacing and Electrophysiology, "Heart rate variability: standards of measurement, physiological interpretation, and clinical use," Circulation, Vol. 93, pp. 1043-1065, 1996. 
[6] Bi-Hua Tan, et al, "Wavelet transform analysis of heart rate variability to assess the autonomic changes associated with spontaneous coronary spasm of variant angina," J Electrovcardiology, Elsevier, Vol.36(2), pp. 117-124, 2003

[7] Taylor J.A and D. L. ECKBERG, "Fundamental relations between short-term R-R interval and arterial pressure oscillations in humans," Circulation, Vol. 93, pp. 1527-1532, 1996.

[8] R. Zhang, K. Iwasaki, J. H. Zuckerman, et al, "Mechanism of blood pressure and R-R variability insights from ganglion blockade in humans," Journal of Physiology, Vol. 543 (1), pp. 337-348, 2002.

[9] R.L.Armentano, A.Simon, J.Levenson, N.P.Chau, J.L.Megnien, and R. Pichel, "Mechanical pressure versus intrinsic effects of hypertension on large arteries in humans," Hypertension, Vol. 18(5), pp. 657-664, 1991.

[10] J.G.Barra, R.L.Armentano, J.Levenson et,al, "Assessment of Smooth Muscle Contribution to Descending Thoracic Aortic Elastic Mechanics in Conscious Dogs," Circ. Res., Vol. 73, pp. 1040-1050, 1993.

[11] J.G.Barra, S.Graf, and R.L.Armentano, "Beneficial Effect of Aortic Smooth Muscle Energy Dissipation in Conscious Dogs," in Proc. World Congress on Medical Physics and Biomedical Engineering, pp. 586, 2003.
[12] N.Saeki, M.Kawamoto, and O.Yuge, "Quantitative view of peripheral circulation," Critical Care Medicine, 28-12, A62(suppl), 2000.

[13] T.Tsuji, P.G.Morasso et al, "Human hand impedance characteristics during maintained posture," Biological Cybernetics, Vol. 72(6), pp.475$485,1995$.

[14] A.Sakane, T.Tsuji, Y.Tanaka, N.Saeki, M.Kawamoto, "Monitoring of Vascular Conditions Using Plethysmogram," The Society of Instrument and Control Engineers, Vol. 40(12), pp. 1236-1242, 2004 (in Japanease)

[15] A.Sakane, T.Tsuji, N.Saeki et al, "Discrimination of Vascular Conditions Using a Probabilistic Neural Network," Journal of Robotics and Mechatronics, Vol. 16(2), pp. 138-145, 2004.

[16] W.W.Nichols, et al, "McDonald's Blood Flow in Arteries: Theoretical Experimental and Clinical Principles, " Arnold, 4th ed, London, 1998.

[17] C.Drott, G.Gothberg, and G.Claes, "Endoscopic transthoracic sympathectomy: an efficient and safe method for the treatment of hyperhidrosis," Journal of the American Academy of Dermatology, Vol. 33(1), pp. 78-81, 1995. 